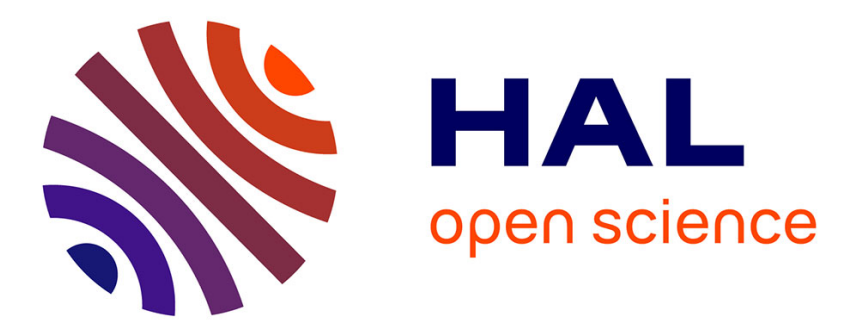

\title{
Couleurs, rituels et normes religieuses en Grèce ancienne Adeline Grand-Clément
}

\section{To cite this version:}

Adeline Grand-Clément. Couleurs, rituels et normes religieuses en Grèce ancienne. Archives de Sciences Sociales des Religions, 2016, 174, pp.127-147. 10.4000/assr.27750 . hal-02177766

\section{HAL Id: hal-02177766 https://hal.science/hal-02177766}

Submitted on 9 Jul 2019

HAL is a multi-disciplinary open access archive for the deposit and dissemination of scientific research documents, whether they are published or not. The documents may come from teaching and research institutions in France or abroad, or from public or private research centers.
L'archive ouverte pluridisciplinaire HAL, est destinée au dépôt et à la diffusion de documents scientifiques de niveau recherche, publiés ou non, émanant des établissements d'enseignement et de recherche français ou étrangers, des laboratoires publics ou privés. 


\title{
COULEURS, RITUELS ET NORMES RELIGIEUSES EN GRÈCE ANCIENNE
}

\author{
Adeline Grand-Clément
}

Éditions de l'EHESS | «Archives de sciences sociales des religions »

2016/2 n 174 | pages 127 à 147

ISSN 0335-5985

Article disponible en ligne à l'adresse :

https://www.cairn.info/revue-archives-de-sciences-sociales-desreligions-2016-2-page-127.htm

Distribution électronique Cairn.info pour Éditions de l'EHESS.

(C) Éditions de l'EHESS. Tous droits réservés pour tous pays.

La reproduction ou représentation de cet article, notamment par photocopie, n'est autorisée que dans les limites des conditions générales d'utilisation du site ou, le cas échéant, des conditions générales de la licence souscrite par votre établissement. Toute autre reproduction ou représentation, en tout ou partie, sous quelque forme et de quelque manière que ce soit, est interdite sauf accord préalable et écrit de l'éditeur, en dehors des cas prévus par la législation en vigueur en France. Il est précisé que son stockage dans une base de données est également interdit. 


\section{Archives de sciences sociales des religions}

174 | Avril-Juin 2016

La force des objets - Matières à expériences

\section{Couleurs, rituels et normes religieuses en Grèce ancienne}

Colors, rituals and religious norms in ancient Greece

Colores, rituales y normas religiosas en la Grecia antigua

\section{Adeline Grand-Clément}

\section{revues.org}

Édition électronique

URL : http://assr.revues.org/27750

DOI : $10.4000 /$ assr. 27750

ISSN : $1777-5825$

\section{Éditeur \\ Éditions de l'EHESS}

\section{Édition imprimée}

Date de publication : 1 avril 2016

Pagination : 127-147

ISBN : $978-2-7132-25-17-8$

ISSN : 0335-5985

Distribution électronique Cairn

\section{CAIRN}

CHERCHER, REPÉRER, AVANCER.

Référence électronique

Adeline Grand-Clément, "Couleurs, rituels et normes religieuses en Grèce ancienne », Archives de sciences sociales des religions [En ligne], 174 | Avril-Juin 2016, mis en ligne le 01 avril 2018, consulté le 10 février 2017. URL : http://assr.revues.org/27750 ; DOI : 10.4000/assr.27750 


\section{Expériences esthétiques}




\section{Adeline Grand-Clément \\ Couleurs, rituels et normes religieuses en Grèce ancienne}

En permettant la production de pigments et de colorants de synthèse, la révolution technologique du XIX ${ }^{\mathrm{e}}$ siècle a entraîné, dans les sociétés européennes, un "désenchantement » de la couleur (Taussig, 2009). Cette dernière a alors perdu le rôle de "substance magique polymorphe " (polymorphous magical substance) qui était le sien jusque-là et qui lui reste dévolu dans nombre de sociétés non occidentales. Les Indiens Chamacoco du Paraguay, par exemple, considèrent que les couleurs exercent une forme de pouvoir sur les objets : elles contraignent ceux-ci à "révéler des significations cachées, significations qui ne sont ni complètes ni durables, sans doute, mais qui peuvent renvoyer, de façon toujours très détournée, à des vérités qui resteraient sinon enfouies »(Escobar, 2007 : 66). Créditées de ce pouvoir de "révélation ", les couleurs peuvent aussi être pensées comme exerçant une influence directe sur le monde sensible, par-delà la sphère du visible: "Étendue sur l'épiderme ou imprégnant un vêtement, la couleur est un instrument symbolique souple, par l'intermédiaire duquel l'être humain peut se situer à l'intérieur du groupe dont il fait partie, mais il peut aussi agir sur l'invisible, et même, selon lui, changer le cours des choses » (Zahan, 1990 : 162). Retrouve-t-on chez les Grecs anciens, qui excellaient eux aussi dans la manipulation des substances colorantes, un rapport analogue à la couleur et à son pouvoir ? Envisageaient-ils les couleurs comme des substances efficaces, activées lors des rituels, et ainsi capables de faire jaillir des vérités cachées, d'investir les objets d'un pouvoir, et d'agir sur le cours des choses ?

Une difficulté de taille se pose à l'helléniste lorsqu'il entreprend de soumettre les matériaux dont il dispose à un tel questionnement. En effet, à la différence de l'anthropologue, l'historien de l'Antiquité ne peut accéder que de manière indirecte aux dispositifs rituels des Grecs. Les traces et les indices qui subsistent dans la documentation figurée et littéraire livrent seulement des représentations, qui doivent être étudiées en tant que telles. Pour mener l'enquête, j'ai donc choisi, à titre exploratoire ${ }^{1}$, de privilégier au sein du corpus de sources écrites celles

1. Précisons d'emblée que les réflexions présentées ici n'ont pas la prétention de proposer une étude exhaustive de la documentation épigraphique disponible : elles visent plutôt à mettre en avant les principales pistes d'analyse qui seront suivies et approfondies, dans le cadre d'un programme de recherches en cours. 
qui pourraient nous sembler de prime abord les plus "directes ", à savoir les inscriptions qui réglementent les actes du culte et les comportements à l'intérieur des sanctuaires. On en connaît aujourd'hui plusieurs centaines, qui proviennent de nombreuses parties du monde grec et s'échelonnent entre le $\mathrm{VI}^{\mathrm{e}}$ siècle avant notre ère et le $\mathrm{III}^{\mathrm{e}}$ siècle de notre ère (Georgoudi, 2010). Les historiens modernes ont donné à ces textes le nom de "lois sacrées », mais la dénomination convient mal, de sorte qu'il faudrait plutôt lui substituer celui de "normes rituelles » (Pirenne-Delforge, Carbon, 2012). Ces textes normatifs, émanant des autorités de la cité ou d'une association cultuelle, étaient gravés sur les murs ou sur des stèles placées bien en vue à l'entrée ou à l'intérieur des sanctuaires. Ils concernent des sujets variés : les exigences de pureté rituelle et les conditions d'accès au sanctuaire, la protection et la gestion des domaines et biens appartenant au dieu, le calendrier du culte et le déroulement des processions et sacrifices, la dévolution des prêtrises... On peut distinguer en fait deux types de réglementation : d'un côté les lois ou décrets ne différant pas des lois et décrets ordinaires, si ce n'est par le fait que les mesures concernent le domaine religieux, de l'autre des conseils à destination du fidèle, qui ne sont pas nécessairement investis d'une valeur juridique (Parker, 2004). En dépit de leurs différences, tous ces textes s'appuient sur une tradition et définissent des normes rituelles ${ }^{2}$, qui nous renseignent sur la façon d'organiser les cultes et de communiquer avec les dieux - même si nous devons avoir à l'esprit que parfois l'inscription omet de rappeler les aspects les plus évidents de la pratique cultuelle qui continuent donc de nous échapper.

Ce vaste corpus, dont la majeure partie a fait l'objet d'éditions commentées (Sokolowski, 1955, 1962, 1969 ; Lupu, 2009)³ , a été étudié sans que les références chromatiques aient été relevées et analysées de manière systématique. Certes, elles ne sont pas légion : dans nombre d'inscriptions on ne trouve pas de précision concernant les couleurs des objets ou substances mobilisés au cours des rituels. Mais il existe quelques cas pour lesquels les Grecs ont jugé bon d'imposer une forme de "norme chromatique ", en prescrivant ou au contraire en proscrivant telle ou telle couleur. Sur le corpus d'environ 420 inscriptions rassemblées par Sokolowski et Lupu, on compte à peine plus d'une trentaine de règlements comportant des notations chromatiques, soit un peu moins de $8 \%$ du total. En dépit de la variété des cas de figure, il me semble que l'on peut dégager quelques pistes d'analyse prometteuses, que je me limiterai ici à présenter, car elles feront l'objet de recherches futures ${ }^{4}$.

2. Sur la norme rituelle comme élément central dans les pratiques religieuses grecques voir notamment Brulé, 2009.

3. Un nouveau projet d'édition, en ligne, est en cours : il reposera sur une redéfinition des contours du corpus (appelé Corpus of Greek Ritual Norms, CGRN) suivant des critères plus sélectifs : Pirenne-Delforge et Carbon, 2012.

4. Dans le cadre d'un programme de recherches mené avec Rendu-Loisel, Synaesthesia. Expérience du divin et polysensorialité dans les mondes anciens. Approche interdisciplinaire et comparée, et financé par l'Idex de Toulouse (2015-2017). 
On constate que la couleur entre en ligne de compte dans trois types de contexte : lorsque la pureté rituelle est en jeu ; lorsqu'il est question des offrandes et en particulier des victimes sacrificielles; lorsqu'il s'agit de pénétrer dans le sanctuaire ou de participer à des cérémonies. Dans ce dernier cas, des codes vestimentaires définissent pour chacun un nuancier de couleurs adapté au domaine du dieu et au temps du rituel. Reprenons successivement chacun des trois types de réglementations cultuelles, pour y traquer les indices du pouvoir que les Grecs ont pu prêter aux couleurs.

\section{Blancheur et pureté rituelle}

Pour pénétrer dans l'enceinte du sanctuaire, espace séparé (temenos) du reste du territoire par la présence de murs ou de bornes, ou pour accomplir une action rituelle, qu'il s'agisse d'une prière, d'un serment ou d'un sacrifice, le dévot grec doit être en état de pureté rituelle (hagneia). Cela signifie être exempt de tout type de souillure, de pollution (miasma), dont les sources sont variées et restent souvent associées au sang versé : en particulier l'accouchement, la mort, le meurtre (Parker, 1983). L'état de pureté exigé - exprimé aussi par l'adjectif katharos (Moulinier, 1952 : 149-170) - concerne donc avant tout l'état physique, mais, à partir du IV e siècle avant notre ère, une attention croissante est portée à la disposition morale du fidèle, au point de faire évoluer les règlements religieux (Chaniotis, 2012). De nombreuses lois témoignent ainsi de ce souci de préserver ou de restaurer la pureté rituelle au sein de l'espace sacré, pour éviter de déclencher la colère divine.

S'il veut se purifier, le fidèle peut utiliser de l'eau ${ }^{5}$ : celle d'une source ou de fontaines et bassins construits à cet effet (Moulinier, 1952: 71-72). Les fouilles archéologiques attestent l'importance de tels dispositifs à l'entrée et à l'intérieur des sanctuaires. Il doit s'agir d'une eau vive, qui jaillit et coule, et non d'une eau stagnante, comme le suggère Hésiode, dans un passage de son poème didactique Les Travaux et les Jours (v. 700 avant notre ère), qui comporte un certain nombre de prescriptions rituelles et morales destinées à son frère Persès :

Que tes pieds ne franchissent pas l'eau qui coule agréablement des fleuves éternels avant d'avoir prié, les yeux tournés vers leur beau cours, tes mains préalablement lavées dans la charmante eau blanche (poluêratô hudati leukô) (Hésiode, Travaux, 737-739).

Pour qualifier l'eau qui sert à la lustration, le poète utilise l'adjectif leukos, "d'un blanc lumineux », un terme qui implique la notion d'éclat et de lumière, souvent associée au soleil et à la joie (Grand-Clément, 2011a : 361-367; Chantraine, 1999 : 632-633). Il veut ainsi suggérer que l'on a affaire à un

5. De nombreuses sociétés attribuent, comme les Grecs, un pouvoir purificateur à l'eau (Simon, $2012: 22$ ). 
liquide clair, frais et limpide - il en souligne d'ailleurs, par un autre adjectif, poluêratos, le caractère désirable. L'accent mis sur la « blancheur » - alors même que l'eau de source peut être, toujours chez Hésiode, qualifiée de " sombre » ou de «violette »- est une manière d'insister sur l'une de ses qualités, à savoir son degré de pureté. En cela, elle s'apparente à l'eau "non mélangée " (akêratos) que Priam utilise dans l'Iliade (XXIV, 303) pour se purifier les mains avant de procéder à des libations et des prières. Mais je crois que la notation chromatique ajoute autre chose : elle donne à percevoir le mode d'action de l'eau, capable d'opérer un blanchiment qui efface toute trace de miasma. Entrant en contact avec les mains, elle agit sur l'ensemble du corps du fidèle et crée les conditions effectives d'une saine communication avec le divin. Signalons aussi que, dans certains rituels grecs, l'eau de mer possède des vertus purificatrices particulières (Parker, 1983 : 226) ; nous pourrions émettre l'hypothèse que, dans ce cas, l'action du sel renforce le pouvoir "blanchisseur" de l'eau.

D'autres produits utilisés dans le cadre des cultes possèdent une vertu analogue aux yeux des Grecs. C'est le cas de l'huile, un produit essentiel à bien des égards, dans une société qui valorise particulièrement les surfaces grasses et luisantes comme signes manifestes de prospérité et de fécondité. On sait que certains rituels d'entretien des statues de culte consistaient précisément à faire briller et à orner les effigies divines (Chankowski, 2014). On recourait pour cela à différents produits, en particulier à de la cire, de la résine, du nitre, de l'huile, des onguents parfumés et des feuilles d'or. Les inscriptions concernant la gestion du sanctuaire de Délos, au cours du III ${ }^{\mathrm{e}}$ siècle avant notre ère, livrent des informations significatives à ce sujet (Homolle, 1890 : 496-503). Une stèle consignant les dépenses de l'année 269 avant notre ère, pour la parure (kosmêsis) de la statue (agalma) de Dionysos, indique que l'huile que l'on a utilisée était « blanche » (elaiou leukou, IG XI, 2, 203, I, 38). La précision chromatique notée dans ce qui était un registre de comptes tenu par les hiéropes, magistrats en charge du culte, manifeste l'attention portée à la «blancheur » comme qualité essentielle du produit utilisé pour le rituel. Le mot souligne que c'est bien une huile purifiée, filtrée, qui doit être utilisée : il s'agit de raviver les couleurs et l'éclat rayonnant (ce que les Grecs appellent la kharis) qui émanent des effigies divines. Signalons que, dans un autre registre, celui des pratiques magiques amoureuses connues grâce à la documentation papyrologique ( $\mathrm{II}^{\mathrm{e}}$ siècle- $\mathrm{V}^{\mathrm{e}}$ siècle avant notre ère), un soin analogue est porté à la qualité de l'huile employée pour remplir les lampes utilisées dans les rituels : là aussi elle doit être "blanche ", c'est-à-dire fraîche, pure et limpide (Zografou, 2010 : 279).

En recourant à des fluides " blancs » et lumineux, les fidèles s'assurent d'une bonne communication avec les puissances divines ${ }^{6}$. On pourrait y ajouter un autre type de substance qui, bien qu'opaque et non transparente, possède un pouvoir analogue. Il s'agit de l'enduit utilisé pour entretenir les autels, dans le

6. Notons que la blancheur est associée à la notion de pureté dans un grand nombre d'aires culturelles (Simon, 2012: 30). 
cadre du culte : les inscriptions stipulent que ces derniers doivent être régulièrement «blanchis » (par exemple une inscription athénienne du IV $\mathrm{e}^{\mathrm{e}}$ siècle concernant les comptes du sanctuaire d'Éleusis, $I G \mathrm{II}^{2}, 1672$, où figure le verbe leukoô, «blanchir»). Reprenons le cas du sanctuaire de Délos : en 278 avant notre ère, les comptes nous apprennent que l'on a utilisé une certaine quantité de "terre blanche" (gê leukê) pour stuquer les autels du Thesmophorion (IG XI, 2, 161, A, 104). Dans ce cas, la blancheur invoquée ne relève pas de la transparence d'un liquide, mais, tout au contraire, de l'opacité d'une matière couvrante. Le point commun entre les deux substances qualifiées de «blanches » réside dans la brillance et l'effet éclaircissant obtenus. Derrière la blancheur, il faut donc imaginer autre chose qu'une simple propriété chromatique : la couleur dit le pouvoir d'action, de transformation de l'eau, de l'huile ou de la terre. De telles substances permettent de raviver l'éclat mais aussi d'uniformiser l'aspect d'une surface, d'effacer ou de masquer les taches et autres marques de souillure sur le corps du fidèle ou sur un objet du culte.

Voilà pourquoi l'exigence de pureté se traduit aussi par le port de vêtements blancs, qui manifestent l'état d'hagneia du dévot. C'est ce dont témoigne une loi gravée au-dessus de l'entrée d'un petit édifice de culte à Priène, au III siècle avant notre ère (Sokolowski, 1955: no 35) : «[...] a obtenu du sort la prêtrise Anaxidêmos, fils d'Apollônios; que l'on pénètre dans [le] sanctuaire (bieron) en état de pureté (hagnon) et revêtu d'un vêtement blanc (esthêti leukêi) ». Le début de l'inscription étant lacunaire, on ignore le nom de la divinité honorée et si l'accès au sanctuaire était public ou réservé au personnel religieux. On sait simplement, grâce à la suite de l'inscription, que le culte était assuré par une association religieuse. Parmi les autres cas analogues qui pourraient être cités, on signalera un règlement de Lindos (île de Rhodes) daté de la même période. Celuici mentionne, parmi une liste de mesures imposées aux fidèles (ne pas porter d'armes, avoir les vêtements propres, ne pas avoir un nœud à la ceinture, ne pas avoir été en contact avec un mort...) le fait de porter des chaussures blanches; si ce n'est pas le cas, le pratiquant doit se déchausser avant de pénétrer dans le sanctuaire (Sokolowski, 1962, no 91).

On est en droit de s'interroger sur le statut du vêtement blanc : est-il simplement là pour signaler l'état de pureté ou est-il la condition même de sa réalisation $^{7}$ ? Pour le dire autrement, est-ce que le tissu communique par capillarité son état de pureté au fidèle, agissant à la manière de l'eau lustrale, ou, pour prendre un exemple issu de l'ethnographie, d'une peinture corporelle lors d'un rite d'initiation ${ }^{8}$ ? Répondre à cette question requiert de préciser au préalable le

7. L'idée selon laquelle l'habit ne fait pas que signaler l'état de pureté mais participe pleinement à sa réalisation et à la construction de l'individu pieux a été mise en avant par de nombreux anthropologues (voir par exemple Mahmood, 2009: 233).

8. Sur les onctions de substances colorées, perçues comme actives et dont l'usage manifeste les liens profonds existant entre esthétique, médecine et magie apotropaïque, voir le cas des Nyangatom d'Éthiopie dans Tornay (2014: 114-115). 
statut symbolique des étoffes blanches dans le monde grec antique : elles représentent des biens de valeur et de prestige. En effet, confectionner et entretenir un tissu immaculé n'était pas aisé, surtout lorsqu'il s'agissait de laine (la fibre la plus utilisée alors). La robe des animaux domestiques étant d'une plus grande variété de couleurs qu'aujourd'hui, les moutons blancs étaient sans doute parmi les plus recherchés. Il fallait sélectionner la meilleure qualité de laine au sein du troupeau, nettoyer la toison, éventuellement la blanchir pour obtenir des fils de la couleur désirée. Une alternative pouvait être de recourir au lin, une fibre végétale permettant de produire des tissus blancs, plus légers - et particulièrement utilisés en Égypte. Ajoutons que, dans un cas comme dans l'autre, conserver l'éclat initial de la teinte et son caractère immaculé supposait un entretien régulier et soigneux du vêtement, ce qui impliquait une certaine aisance sociale. Voilà pourquoi une étoffe blanche était surtout de mise lors des fêtes ou des grandes occasions ; elle suscitait de l'admiration, attirait les regards et permettait de distinguer l'élite 9 . L'idéal recherché était sans doute celui que l'on trouve exprimé dans l'Iliade, à propos du voile d'Héra : décidée à séduire son époux, cette dernière se couvre d'un peplos «blanc (leukos) comme un soleil » (Iliade, XIV, 185). La blancheur évoquée par le poète traduit l'éclat, la kharis qui se dégagent du vêtement radieux endossé par la déesse.

Rappelons en outre que le vêtement blanc s'oppose au vêtement teint (Gerschel, 1966). Or l'un des verbes servant à désigner l'acte de teindre est miainô, un mot qui signifie aussi «souiller ». En effet, les Grecs, dans certains contextes, envisagent la coloration comme une tache, un masque, qui fait écran, recouvre et dénature. En recourant à un vêtement blanc, c'est-à-dire non teint, le fidèle de Priène se présente sans fard devant la divinité - tout comme, dans d'autres sanctuaires, il doit enlever ses chaussures et se dépouiller de tout ornement. On sait que le culte des dieux égyptiens - qui disposent d'ailleurs d'un sanctuaire à Priène, à la fin du $\mathrm{III}^{\mathrm{e}}$ siècle avant notre ère - requérait souvent un tel costume, généralement en lin : selon Plutarque (Isis et Osiris, 4), une telle prédilection des Égyptiens pour cette fibre végétale, préférée à la laine, s'expliquait par une exigence de pureté et de piété.

Chez les Grecs, les tissus blancs ont aussi le pouvoir d'attirer l'attention des dieux et de susciter leur bienveillance. Voilà pourquoi les suppliants qui demandent asile et protection dans un sanctuaire portent à la main des bandelettes de laine blanche, enroulées sur des bâtons ou des rameaux végétaux ${ }^{10}$. Le poète tragique Eschyle ( $\mathrm{V}^{\mathrm{e}}$ siècle avant notre ère) dépeint les Danaïdes, réfugiées autour de l'autel d'Argos, comme étant leukostrepheis, c'est-à-dire tenant à la

9. Sur la difficulté à fabriquer et à entretenir des vêtements réellement blancs, qui perdure au Moyen Âge, voir Pastoureau (1997: 68-70).

10. Sur le rôle des rameaux végétaux, souvent une branche d'olivier, voir Naiden (2006: 56-57) : l'auteur estime que les bandelettes de laine qui peuvent être enroulées expriment l'idée de pacifisme, car elles sont associées au travail féminin de tissage. 
main des rameaux aux blanches guirlandes (Suppliantes, 191-192 et 334). Ce n'est pas tant la présence de bandelettes de laine que leur couleur immaculée et éclatante qui renforce l'efficacité de la prière aux divinités. Dans une autre pièce, les Euménides, c'est Oreste le matricide qui, pourchassé par les Érinyes, pénètre dans le sanctuaire d'Apollon, implorant le pardon et la protection du dieu. Il serre dans sa main une toison "d'un blanc éclatant» (argêti, Euménides, 45) ${ }^{11}$. Ici, la blancheur possède une double signification : non seulement elle appuie la demande de protection, mais elle intervient de manière active dans le processus de purification nécessaire après le meurtre.

Ainsi, lorsqu'il s'agit d'entrer en communication avec les dieux, des matières ou objets blancs peuvent être privilégiés. Il serait pour autant abusif de laisser entendre qu'ils auraient l'apanage du pouvoir purificateur : on sait par exemple que le sang sacrificiel peut posséder une efficacité analogue dans certains rituels cathartiques (Moulinier, 1952 : 89-91 ; Parker, 1983 : 371-373) et lors des sacrifices, lorsqu'on en arrose l'autel. De nombreuses images sur les vases en témoignent : les traces de sang séché sur l'autel, que les peintres signalent par des rehauts de couleur rouge ou pourpre, sont la marque tangible de la piété des hommes, car elles révèlent que les cultes se déroulent de manière régulière et en conformité avec les normes rituelles (van Straten, 1995 : 104).

\section{Couleurs et victimes sacrificielles}

Le sacrifice sanglant (la thusia) occupe une place centrale dans la religion grecque : la mise à mort de l'animal et surtout le partage des viandes qui s'ensuit instaurent une relation à la fois verticale (entre hommes et dieux) et horizontale (au sein de la communauté de fidèles). C’est à Prométhée que les Grecs attribuent la paternité de ce rite, qui consiste à séparer la part des hommes de celle des dieux. Cette dernière est constituée de ces os blancs enrobés de graisse luisante qui sont brûlés sur l'autel et dont l'odeur nourrit les Immortels (Detienne, Vernant, 1979 ; Brulé, Mehl, 2008).

Le rituel fait l'objet d'un encadrement normatif. Un certain nombre de lois sacrées précisent les conditions requises dans le choix de la victime animale. Il existe même dans certaines cités une procédure spécifique, la dokimasie, qui consiste en un examen préalable des bêtes par les magistrats ou les prêtres, pour vérifier leur conformité par rapport aux exigences du culte (Gauthier, 1984 ; Feyel, 2006). La plupart du temps, il s'agit de se procurer des animaux qualifiés de «beaux " et de "sains ", "intègres " (holoklaron), c'est-à-dire dépourvus de malformation. Il faut que la bête plaise à la divinité, afin que cette dernière accepte le sacrifice, et pour cela, on la pare (Brulé, Touzé, 2008). On peut

11. Sur cet adjectif, associé à leukos, voir Grand-Clément (2011a : 364-365). 
aller jusqu'à dorer les cornes d'une vache, comme c'est le cas dans l'Odyssée, lorsque Nestor, roi de Pylos, souhaite s'attirer les faveurs d'Athéna (Odyssée, III, 438) - une pratique confirmée du reste par l'épigraphie délienne d'époque hellénistique ${ }^{12}$.

La documentation épigraphique nous renseigne sur les différents paramètres pris en compte pour choisir l'animal. Les inscriptions précisent ainsi le type d'animal (le plus souvent un ovin, un porcin ou un bovin), son âge, son sexe, et parfois sa couleur, qui peut être évoquée soit par une formule générale - la bête doit être eukhrous, c'est-à-dire d' " une belle couleur »-soit par un adjectif renvoyant à une nuance déterminée. Dans le premier cas, on insiste sur l'absence de tache : la plénitude de la couleur du pelage et son uniformité sont gages de bonne santé et de beauté, des critères essentiels dans le cadre du culte rendu aux dieux (Georgoudi, 2007 ; Feyel, 2006 : 36-42). Mais que dire lorsque le choix d'une couleur particulière est spécifié ? On peut émettre l'hypothèse que les propriétés chromatiques requises pour les animaux sont censées renforcer le lien établi avec la puissance destinataire, parce qu'elles ont été choisies en accord avec leur domaine d'intervention spécifique, afin de garantir l'efficacité du rituel. Cependant, S. Georgoudi a raison d'inciter à la prudence lorsqu'il s'agit d'interpréter les intentions des fidèles, tant la documentation épigraphique révèle l'extrême diversité des traditions cultuelles à l'intérieur du monde grec : "Chaque cité informe son propre panthéon, ses puissances divines particulières, selon un mode hiérarchique propre à son histoire et à ses traditions, mais également à ses capacités financières. "(Georgoudi, 2007 : 42). L'auteur identifie plusieurs facteurs susceptibles de jouer : le contexte cultuel local, la personnalité du destinataire du sacrifice, les contraintes budgétaires et considérations économiques, l'écosystème et les pratiques d'élevage de la région concernée, les enjeux sociopolitiques et les questions de prestige au sein de la communauté.

Ce sont les calendriers cultuels, enregistrant la liste des fêtes célébrées par la cité au cours de l'année, qui fournissent le plus d'éléments d'information. Trois teintes peuvent être prescrites pour l'animal sacrifié : le blanc (leukon), le noir (melan) et, moins fréquemment, le roux/ rouge feu (eruthros, purrhos). On retrouve une telle triade chromatique dans d'autres aires culturelles - ce qui n'a pas manqué d'alimenter le mirage d'un universalisme de la valeur symbolique de ces trois couleurs en contexte rituel ${ }^{13}$. Pourtant, les configurations sont variées

12. Cf. les comptes de l'amphictyonie des années 370 avant notre ère : $I G I^{2} 1635,35-37$.

13. L'une des études de référence sur cette question du symbolisme de la triade noir/blanc/ rouge est celle de Victor Turner sur les rituels ndembu (Afrique centrale). L'anthropologue montre que les couleurs sont conçues comme des flots émanant de la divinité et chargés d'un pouvoir, qu'elles communiquent à l'ensemble des éléments du monde La manipulation de substances rouges, blanches, et plus rarement noires, lors des rites d'initiation, prend sens à la lumière de la cosmologie ndembu, qui repose sur le "mystère " (mpangu) des trois rivières primordiales, celles de la blancheur, de la rougeur et de la noirceur (Turner, 1966). Citons en outre, parmi les autres études de référence, Jacobson-Widding (1979), qui concerne le Congo. 
d'une aire culturelle à l'autre. En Inde, par exemple, les Santal ajoutent au noir, au blanc et au rouge de leurs victimes sacrificielles le bigarré et l'inversé, en particulier pour les volailles qui constituent l'offrande la plus courante (CarrinBouez, 1978). De nombreux paramètres doivent être pris en compte si l'on cherche à interpréter le sens accordé à la couleur prescrite pour l'animal. En effet, Marine Carrin-Bouez a montré qu'il n'existe pas de système symbolique générique et fixe, mais plutôt des valeurs positionnelles, relatives, attribuées à la coloration des robes et plumages des bêtes. Les couleurs réagissent les unes par rapport aux autres. C'est d'ailleurs ainsi que certains chercheurs ont proposé d'expliquer l'alternative blanc/noir que l'on rencontre en Grèce. Elle correspondrait selon eux à une polarité entre deux catégories de dieux : les ouraniens d'un côté, les chtoniens de l'autre, auxquels seraient associés les héros et les défunts (Sokolowski, 1955 : 117-118). Aux premiers les Grecs destineraient plutôt des animaux blancs, aux seconds des animaux noirs, le plus souvent sacrifiés la nuit (Stratiki, 2004). Pour corroborer cette hypothèse, on convoque souvent un passage du chant III de l'Iliade, dans lequel une telle bipartition semble effectivement fonctionner. Au moment de sceller un accord, Achéens et Troyens prennent les dieux à témoin et procèdent à des sacrifices :

Apportez deux agneaux, agneau blanc (leukon) et agnelle noire (melainan), pour la Terre (Gê) et pour le Soleil (Hélios). Nous en apporterons, nous, un autre pour Zeus (Iliade, III, 103-104).

On constate ici que le même type d'animal est choisi pour Gê et pour Hélios, mais que les ovins se distinguent par leur sexe et par leur couleur. La blancheur pour le Soleil, la noirceur pour la Terre : voilà qui paraît correspondre à l'opposition ouranien/chtonien. Pour autant, le système n'est pas binaire : pour Zeus, l'Olympien par excellence, qui règne sur le ciel, un troisième ovin est requis, et rien n'est dit de sa couleur.

En fait, si la grille d'interprétation blanc=ouranien et noir=chtonien peut convenir dans certains cas, elle est simpliste et réductrice ${ }^{14}$. Elle ne convient pas à la plasticité du polythéisme grec soulignée plus haut : chaque cité possède son panthéon local, composé de divinités dont les compétences sont précisées par des épiclèses. Il existe de fait une infinité de variations dans les pratiques sacrificielles des Grecs. C’est donc dans les spécificités des configurations panthéoniques locales et dans le pouvoir prêté à telle ou telle puissance divine qu'il faut rechercher une explication possible à la nature de la couleur prescrite (Lupu, 2005 : 141). Ajoutons, pour complexifier le tableau et montrer à quel point la prudence doit être de mise, qu'il existe des cas où deux couleurs sont possibles. Le calendrier sacrificiel du dème attique de Thorikos, daté de 440-420 avant notre ère, indique qu'à deux reprises dans l'année les Athéniens sélectionnent

14. Sur la nécessité de prendre un peu de distance avec la distinction entre dieux olympiens et dieux chthoniens, voir la mise au point dans Pirenne-Delforge (2008 : 181-183). 
pour Dionysos une victime qui est soit rousse (purrhon), soit noire (melana) : il s'agit d'une chèvre au mois d'Anthesterion, puis d'un bouc au mois de Mounichion (Lupu, 2005, no 1, 34 et 45-46). On observe un cas analogue dans la cité de Camiros, au III ${ }^{\mathrm{e}}$ siècle avant notre ère : à Hélios (le Soleil), divinité majeure de l'île de Rhodes, on doit sacrifier un bœuf blanc ou roux (Sokolowski, 1962, no 97, 2-4). On peut supposer que les dévots grecs faisaient preuve de pragmatisme en accomplissant les rituels : ils devaient probablement négocier avec les prescriptions et conserver une certaine marge de liberté dans le choix de la couleur de la victime, même lorsque telle ou telle couleur était attendue, comme c'est le cas chez les Nyangatom d'Éthiopie étudiés par Serge Tornay (2014: 116-125).

Arrêtons-nous sur un exemple précis, celui de la cité de Mykonos (Cyclades) à l'époque hellénistique. Le calendrier cultuel, connu par une stèle de marbre gravée vers 200 avant notre ère (Sokolowski, 1969, nº 96), a été aménagé à la suite d'un changement politique intervenu dans l'île : les différentes communautés ont été réunies pour former une seule et même cité. Pour sceller le synœcisme, l'assemblée des citoyens a donc établi un calendrier commun, fondé sur la reprise de fêtes existantes, mais aussi la création de nouvelles célébrations :

Dieux ! À la bonne fortune! Sous l'archontat de Kratinos, Polyzêlos et Philophrôn, lorsque les cités (de l'île) se réunirent par synœcisme, il a paru bon aux citoyens de Mykonos d'offrir les sacrifices suivants en plus de ceux accomplis auparavant et de rectifier ces derniers :

le 12 du mois de Posideôn, à Poséidon Temenitês un très beau bélier blanc entier (krios kallisteuôn leukos enorkhês); le bélier ne sera pas emmené dans la ville; on découpera son dos et son omoplate; l'omoplate sera consacrée par une libation; au prêtre la langue et l'épaule; le même jour à Poséidon Phukios un agneau blanc entier (amnos leukos enorkhês) ; interdit aux femmes ; que le Conseil achète les victimes en versant vingt drachmes prises sur l'impôt des poissons ; le même jour à Déméter Khloê deux truies des plus belles dont une, pleine; le dos de celle qui est pleine sera découpé ; que le Conseil sélectionne les truies; que les archontes donnent au mageiros une hanche et une cuisse de l'autre truie ; deux chénices d'orge, trois cotyles de vin ;

le 10 du mois de Lênaiôn, au son d'un hymne pour les fruits de la terre, une truie grosse pour la première fois à Déméter, pour Korê un sanglier adulte et pour Zeus Bouleus un porc; que pour ces sacrifices les hiéropes prennent sur les fonds sacrés et qu'ils fournissent bois et grains d'orge ; que les archontes et les prêtres veillent à la qualité des victimes; s'il est besoin de quelque chose pour sacrifier sous d'heureux auspices, que les hiéropes les procurent ; qu'aille à la fête celle qui le veut des citoyennes de Mykonos comme des habitantes de l'île initiées aux Mystères de Déméter ;

le 11, à Tapaêthos (?) pour Sémélé le sacrifice annuel ; la neuvième part sera brûlée ; le 12, à Dionysos Lenaios, le sacrifice annuel; pour les fruits de la terre à Zeus Khthonios et à Gê Khthonia, des victimes noires (melana), écorchables, d'un an ; interdit aux étrangers; les participants au sacrifice prendront leur repas sur place;

le 10 du mois de Bakkhiôn, à Deiras, pour Dionysos Bakkheos un jeune chevreau des plus beaux; que les hiéropes en paient le prix et qu'ils participent au repas que l'on fera sur place ; le 7 du mois d'Hékatombaiôn, à Apollon Hekatombios un taureau et dix jeunes agneaux, le dos du taureau sera découpé ; on donnera au prêtre la langue 
et une épaule du taureau; sur les agneaux que les enfants offrent en sacrifice, on prendra pour le prêtre une langue et pour chaque enfant une langue ; sur les agneaux sacrifiés par les couples de jeunes mariés, on prendra une langue pour le prêtre et une langue pour chacun des deux couples de jeunes mariés; le même jour à Akhélôos, une victime adulte et dix agneaux ; trois de ces bêtes, l'animal adulte et deux autres, seront immolées sur l'autel, les bêtes restantes seront jetées dans le fleuve; celui qui cultive la terre située à Sa [...], appartenant à Akhélôos, versera son loyer pour les sacrifices habituels à Akhélôos ;

le 15 à Arkhegetês, le sacrifice annuel ${ }^{15}$...

Si la fin de l'inscription est mutilée, le texte est suffisamment long pour offrir une bonne vision du déroulement des différentes fêtes religieuses programmées au cours de l'année et des sacrifices sanglants auxquels elles donnent lieu. Il faut cependant garder à l'esprit que, comme le précise l'intitulé, la stèle ne reprend pas l'intégralité du calendrier cultuel, mais indique seulement ce qui a changé et doit désormais être commun à l'ensemble des membres de la nouvelle communauté. La première remarque qui s'impose est que, sur la dizaine de cas de sacrifices sanglants mentionnés, seuls trois s'accompagnent d'une prescription relative à la couleur de l'animal : deux fois pour Poséidon (victimes blanches), une fois pour Zeus et Gê (victimes noires).

Considérons d'abord le cas de Poséidon. Ce dieu occupe une position prééminente dans le calendrier : c'est la divinité qui a donné son nom au mois qui marque le début de l'année ${ }^{16}$. La couleur blanche requise pour l'agneau et le bélier qui lui sont destinés pourrait surprendre de prime abord, si l'on en reste à la distinction olympien/chtonien évoquée plus haut. On attendrait en effet de ce maître des profondeurs marines, "l'Ébranleur de la terre » chez Homère, des victimes plutôt sombres. C'est d'ailleurs le cas dans l'Odyssée (III, 6) : les Pyliens lui sacrifient sur la grève des taureaux d'un noir immaculé (pammelanas). Pindare raconte que les Argonautes, en arrivant en Thrace, lui offrent un troupeau roux (phoinix), qui se trouvait à proximité du rivage (Pythiques, IV, 205). Alors pourquoi, à Mykonos, les victimes doivent-elles être blanches? Le choix d'ovins plutôt que de bœufs ou de taureaux (que les habitants de Mykonos réservent à Apollon, lors du mois d'Hekatombaiôn) témoigne du souci d'avoir des animaux blancs à offrir à Poséidon.

L'explication réside sans doute dans les spécificités du panthéon local, mais malheureusement on ne le connaît pas bien. L'inscription révèle que le dieu est vénéré dans le cadre d'au moins deux cultes distincts, correspondant à deux épiclèses : le Poséidon du téménos (= l'enceinte sacrée du sanctuaire) et celui du phukos, une variété d'algue, qui tire son nom du colorant rouge qu'elle fournissait et qui servait de produit cosmétique. Le premier cas associe le dieu à

15. Traduction Le Guen-Pollet (1991 : 191-192) légèrement modifiée.

16. Il est difficile de déterminer la position des mois cités dans le texte, car chaque cité grecque possédait son propre calendrier, et celui de Mykonos est moins connu que celui d'autres cités comme Athènes. 
l'enceinte sacrée du sanctuaire, tandis que le second le qualifie comme dieu protecteur de la pêche - une activité vitale et lucrative pour les habitants de l'île, sans aucun doute, grâce au commerce des algues, du poisson ou des coquillages producteurs de pourpre (Zoumbaki, 2014 : 329). Une explication rapide pourrait consister à relier la couleur des offrandes sacrificielles à celle du domaine d'intervention du dieu, à savoir la mer, que les poètes qualifient volontiers de «blanche ", car elle regorge de sel et s'ourle d'écume. Mais il me semble que la raison doit plutôt être cherchée ailleurs. On constate en effet que le même jour, un troisième sacrifice a lieu, en l'honneur de Déméter. Son épiclèse, Khloê, littéralement " celle qui verdit ${ }^{17}$ ", suggère le lien avec le renouveau de la végétation : tout cela renvoie au retour du printemps. Le choix de deux truies particulièrement «belles ", dont l'une doit être pleine, traduit bien la volonté de garantir la fécondité de l'année qui commence et des récoltes à venir. Or cette saison coïncide avec la réouverture de la navigation en Méditerranée, après la trêve hivernale. La blancheur des victimes offertes à Poséidon va donc dans le même sens : elle a une valeur propitiatoire, qui repose sur le lien que les Grecs établissaient entre cette couleur et la notion de fécondité. En sacrifiant des victimes blanches, la communauté de Mykonos espère bénéficier d'une année florissante, à tous points de vue : les ressources de la terre et celles de la mer seront mises à profit pour faire croître la prospérité du temenos du dieu et, partant, celle de la communauté qu'il protège ${ }^{18}$.

Considérons à présent le cas de Zeus, une divinité concernée par au moins deux sacrifices qui se déroulent au cours du mois de Lénaiôn, à seulement deux jours d'intervalle. La première fois, c'est en tant que Bouleus qu'on lui destine un porc, au cours d'une fête qui concerne aussi Déméter et Korê, et implique l'ensemble de la composante féminine de l'île (on songe alors à des fêtes se rapprochant des Thesmophories $\left.{ }^{19}\right)$. Rien n'est spécifié au sujet de l'animal, si ce n'est qu'il doit faire l'objet d'un examen par les archontes et les prêtres, afin de répondre aux critères de beauté habituels. L'important est qu'il s'agisse d'un porc. Dans le second cas, au contraire, ce n'est pas l'espèce qui compte mais la couleur des victimes sacrificielles : il est stipulé qu'elles doivent être noires. La couleur prescrite renvoie alors au domaine agricole et rattache Zeus, comme sa parèdre Gaia (Terre), avec qui il partage les victimes sacrificielles et surtout la

17. L'épiclèse est attestée chez Pausanias (I, 22, 30). Sur le sens de l'adjectif khlôros, qui renvoie à la notion de viridité, voir notamment Irwin, 1974 : 31-78; il s'agit de la couleur des jeunes pousses et de l'immaturité végétale (Dimakopoulou, 2010 : 94-97).

18. On pourrait à cet égard suggérer un rapprochement avec le calendrier cultuel de Milet, gravé vers 500 avant notre ère, qui stipule qu'il faut offrir une brebis blanche (leukè) et pleine à Héra Antheê, la "fleurie/florissante ", une déesse chargée ici du renouvellement des forces vives de la cité (Sokolowski, 1955 : n 41, 6).

19. Les Thesmophories, qui existent dans de nombreuses cités grecques, sont des fêtes religieuses en l'honneur de la déesse liée au monde agraire, Déméter. Elles ont la particularité d'être prises en charge par les épouses des citoyens et visent à garantir la prospérité des récoltes et la fécondité des femmes. 
même épiclèse, au monde chtonien. Appliquée à la terre, la noirceur en souligne le caractère fécond (Grand-Clément, 2011a : 356-357). De plus, il est précisé que c'est « pour les fruits de la terre » que le rituel a lieu : en ancrant l'Olympien dans les champs de la nouvelle cité, les habitants de Mykonos cherchent à garantir la pérennité du lien qui unit la nouvelle communauté et à s'assurer de la fertilité des champs qui serviront à faire fructifier le corps civique.

Venons-en à présent aux cas, plus rares, de victimes sacrificielles « rouges/ rousses » (eruthros, purrhos) - qui, nous l'avons vu, sont parfois interchangeables avec des victimes noires ou blanches. Prenons l'exemple des rites cathartiques de Cyrène (Libye), connus par une inscription datée de la toute fin du IV siècle avant notre ère (Sokolowski, 1962, $\mathrm{n}^{\mathrm{o}} 115$ ). Elle contient une liste de prescriptions dont les habitants attribuaient la paternité à l'oracle d'Apollon (à Delphes), comme le stipule l'intitulé : "Apollon a décrété que les Cyrénéens devraient vivre en Libye en observant les purifications et les abstinences et [...] ». En fait, le code de lois renferme une compilation de réglementations très diverses, issues de périodes différentes (Parker, 1983 : 334-335). On y trouve en particulier des règles liées à différents états pouvant induire des formes de miasma (relations sexuelles, accouchement...). La première d'entre elles évoque l'attitude à avoir si un fléau menace la communauté :

"Si la maladie ou [...] la mort advient contre le pays ou la cité, il faut sacrifier devant les portes et l'apotropaion à Apollon Apotropaios un chevreau rouge (khimaron eruthron). "

On connaît les compétences d'Apollon en matière d'épidémie. Lorsqu'il est en colère, le dieu archer peut déclencher de terribles maladies par ses flèches, comme c'est le cas au début de l'Iliade; apaisé, en revanche, il peut y mettre un terme. Mais pourquoi faut-il un chevreau roux pour l'engager à agir dans ce sens ? Il ne s'agit pas ici de plaire particulièrement au dieu, mais plutôt d'identifier une victime susceptible d'incarner la source du mal, de se charger de la souillure que l'on cherche à expulser; dans ce cas le choix d'un animal comme le chevreau n'est sans doute pas anodin (Parker, 1983 : 334). Il joue le rôle de bouc émissaire, que les Grecs nommaient pharmakos, une victime expiatoire, et la localisation du rituel à l'extérieur de l'enceinte, devant les portes, renforce le caractère apotropaïque de la procédure. On agit ici dans l'urgence, et non dans le cadre d'un calendrier cultuel régulier, comme à Mykonos, ou à Thorikos, lorsque l'on choisit chaque année une victime noire ou rousse pour Dionysos.

Il me semble que la rousseur participe bien de l'efficacité du rituel cathartique : une telle couleur possède une connotation qui peut s'avérer négative, ou du moins inquiétante dans le système de représentation des Grecs, car elle implique une forme de marginalité. Les pilosités rousses sont souvent la marque de l'Autre : chevelures rousses des barbares du nord, les farouches Thraces, et pelages fauves des bêtes sauvages (Grand-Clément, 2011a : 259-262). La rousseur du chevreau contribue donc ici à matérialiser le miasma, qu'il s'agit de 
repousser. La proximité de Cyrène avec l'Égypte peut également jouer : on sait que dans ce pays, les êtres (hommes et animaux) roux sont considérés comme violents et sanguins, et associés au dieu Seth ${ }^{20}$.

Il est possible de proposer un rapprochement avec des cas mentionnés dans les poèmes du début $d u V^{e}$ siècle avant notre ère : on constate que les victimes rousses y semblent surtout destinées à Artémis, déesse de la chasse, des confins et des situations guerrières extrêmes. Le terme employé par les poètes pour évoquer cette "couleur de feu " est alors phoinix, un rouge brun sombre, associé à la teinture écarlate (Grand-Clément, 2011a : 350-352). Dans une ode de Bacchylide (Épinicies, V, 57), il est question d'un sacrifice de chèvres et de bœufs " au dos roux" (phoinikonôtôn), qui ne suffit cependant pas à apaiser la fureur de la déesse. Cette dernière déchaîne en effet contre les hommes un véritable fléau: le sanglier de Calydon. Et dans un autre poème (Épinicies, XI, 69), Proitos, lorsqu'il implore Artémis de rendre la raison à ses filles, en proie à la folie, promet de lui immoler "vingt bœufs au poil roux, qui ignorent le joug " (phoinikotrikhas).

Il apparaît donc que, lors des sacrifices animaux, le critère chromatique n'est pas toujours pris en compte; lorsque c'est le cas, il n'a pas de signification prédéterminée : c'est au cours de la procédure rituelle que l'efficacité de la couleur trouve à s'exprimer. Elle n'est alors que l'un des éléments se combinant avec d'autres pour permettre de sélectionner la victime susceptible d'être acceptée par la divinité concernée par le sacrifice, en accord avec sa sphère d'intervention, son mode d'action, mais aussi sa place au sein du panthéon local. Les fidèles souhaitent que la victime plaise à la puissance invoquée ou lui ressemble : dans ce cas, la couleur contribue à attirer ses faveurs, mais il existe aussi des cas où il s'agit d'expulser une source de pollution, que la couleur incarne et concentre.

\section{Des couleurs liturgiques?}

La couleur et le décor du vêtement de l'officiant, des participants aux rites et des dévots entrant dans le sanctuaire, font dans certains cas l'objet d'une réglementation plus ou moins stricte. Cette dernière tient compte des exigences de pureté rituelle dont nous avons parlé précédemment, mais est également soustendue par d'autres préoccupations.

Prenons le cas des prêtres. Leur fonction est parfois - mais pas systématiquement - associée au port d'un costume distinctif. Ce dernier varie suivant les cités et les cultes, mais il se caractérise souvent par la combinaison de deux couleurs : le blanc et le pourpre/l'écarlate (Jones, 1999). Ainsi dans la cité de Pergame (Asie mineure), une inscription d'époque hellénistique a conservé la lettre d'un roi

20. Cf. Diodore, I, 88 : l'auteur mentionne les bœufs roux, purrhous bous, que l'on sacrifie à Typhon-Seth (voir Volokhine, 2010). 
attalide définissant les conditions d'exercice d'une prêtrise récemment créée (Sokolowski, 1955 : no 11) :

[...] que [le prêtre] désigné (à son) tour par le sort porte une chlamyde blanche (leukèn) et une couronne d'olivier avec une bandelette écarlate (phoinikiou); qu'il prenne en guise de geras des bêtes sacrifiées, la peau, une cuisse, et le revenu des boutiques que j'ai consacrées; que le prêtre désigné à son tour par le sort les loue et qu'à sa sortie de charge il les transmette réparées à son successeur, sous peine de payer la dépense entraînée par la réparation; qu'il soit exempté de toutes les liturgies, le temps pendant lequel il portera la couronne; qu'il conserve les objets en argent du dieu et les autres offrandes et qu'il les remette à son successeur. Porte-toi bien !

La lacune initiale ne permet malheureusement pas de déterminer à quelle divinité se rapporte la prêtrise. Les conditions de dévolution (tirage au sort) et d'exercice de la fonction (limitation dans le temps, obligations financières et privilèges) montrent que la prêtrise ressemble fort à une magistrature ordinaire. Une différence importante réside cependant dans le port d'un costume distinctif, qui a pour fonction de signaler dans l'espace public le rôle qu'il joue au service de la communauté. On sait que dans cette même cité de Pergame, tous les prêtres n'étaient pas tenus de se conformer à une norme vestimentaire : une autre inscription (Sokolowski, $1953: \mathrm{n}^{\circ}$ 37) révèle en effet que le prêtre de Dionysos Phleos pouvait porter le vêtement de son choix ; on le reconnaissait seulement au port d'un bandeau de lierre doré. Le prêtre du culte institué par le roi attalide, lui, est revêtu d'un manteau (chlamyde) d'un blanc éclatant et a la tête ceinte d'une couronne végétale contenant un tissu écarlate. C'est cette dernière couleur surtout qui signale l'exemption de liturgie au cours de l'année, comme le suggère le texte. Quant au vêtement leukos, doit-il être porté quotidiennement ou seulement lors de l'accomplissement des rituels ? C'est difficile à dire. Et quelle est sa fonction ? S'agit-il simplement de distinguer le prêtre du reste de la communauté ou de rendre manifeste l'état de pureté qui doit être le sien durant le temps où il exerce la prêtrise? Allons plus loin : est-ce une manière de lui rappeler cette exigence, ou la condition qui la rend possible et effective ? Cette blancheur contribue-t-elle à le rendre apte à diriger les opérations rituelles ?

Si l'on ne peut trancher dans le cas de Pergame, on dispose d'un autre témoignage, littéraire cette fois-ci, qui présente un cas où la norme rituelle impose au prêtre de changer de costume, pendant un temps donné. Plutarque (Vie d'Aristide, 21, 1) décrit le sacrifice institué après la victoire de Platées contre les Perses, lors de la fête des Eleutheria, en l'honneur de Zeus Eleutherios ( Libérateur »), qui se déroulait à la fin du mois de novembre. La précision avec laquelle l'auteur, originaire de la région béotienne, détaille le déroulement des opérations et l'ambiance sensorielle ainsi créée laisse penser qu'il a assisté personnellement à l'une de ces célébrations. Son témoignage est d'autant plus digne d'intérêt qu'il a lui-même exercé des fonctions sacerdotales à Delphes, et accorde donc une grande attention aux procédures rituelles : 
Le seize du mois de Maïmactériôn, les Platéens organisent une procession qui commence au point du jour. Un joueur de trompette ouvre la marche en sonnant le signal du combat. Derrière lui, s'avancent des chariots remplis de myrte et de couronnes, puis un taureau noir (melas) et des jeunes gens qui portent dans des amphores des libations de vin et de lait, ainsi que des cruches d'huile et de parfum. Ces jeunes gens sont de condition libre : aucun esclave n'a le droit de participer à ce service, étant donné que c'est pour la liberté que ces guerriers sont morts. Vient enfin l'archonte de Platées : d'ordinaire, il lui est interdit de toucher du fer, et il ne doit porter que des vêtements blancs (leukês), mais ce jour-là, il est vêtu d'un vêtement écarlate (phoinikoun), il tient une hydrie prise au dépôt des archives et, l'épée à la main, il traverse la cité pour se rendre devant les tombeaux. Là, il prend de l'eau à la source, lave de ses propres mains les stèles, les oint de parfum, égorge le taureau sur le bûcher et, après avoir prié Zeus et Hermès Khthonios, invite les nobles héros qui sont morts pour la Grèce à venir participer à ce repas et à se gorger de sang. Puis, procédant au mélange du vin dans le cratère, il le répand en disant : "J'offre à boire aux guerriers qui sont morts pour la liberté des Grecs ». Cette cérémonie est encore célébrée de nos jours par les Platéens.

Lorsque l'on examine l'ensemble de la séquence rituelle, on constate qu'elle diffère de la procédure mise en œuvre pour la thusia ordinaire, comme le suggère d'emblée le choix de la trompette, arme évoquant la guerre, en lieu et place de l'aulos, la double-flûte utilisée pour accompagner les processions sacrificielles. La fête des Eleutheria célèbre la victoire obtenue sur l'envahisseur perse, et donc la libération du territoire, mais commémore aussi les soldats-citoyens défunts qui sont tombés pour défendre leur mère patrie et ont été enterrés sur place. Le rituel s'adresse donc à des divinités au caractère chtonien fortement marqué, comme le souligne l'épiclèse ; Hermès permet d'entrer en communication avec le monde des morts. Le choix d'un taureau noir va dans le même sens. Le sang versé vise à nourrir les défunts, qui viennent boire - la parole du prêtre insiste sur ce point : l'offrande de sang, qui porte le nom d'haimakouria et est prolongée par la libation de vin, vise surtout à solliciter la participation des défunts (Pirenne-Delforge, 2008 : 210). Un tel acte rituel rappelle fort ce que l'on trouve dans le passage de l'Odyssée appelé la Nekuia, au moment où Ulysse invoque Tirésias et l'âme des défunts après avoir sacrifié des animaux noirs et répandu le sang «aux sombres nuances »(kelainephes).

Pour célébrer la fête en l'honneur des héros tombés au combat, fête qui conjugue des aspects militaires et chtoniens, le prêtre doit changer de vêtement. Il doit arborer alors une phoinikis, un vêtement qui est d'ordinaire celui des soldats ou des chefs d'armée. La tunique écarlate se charge de signification en ce qu'elle s'oppose à la blancheur de son costume habituel (Gernet, 1957). Mais elle n'agit pas comme simple signe destiné à marquer le caractère martial de cette cérémonie : il me semble en effet que la tunique écarlate, tout comme l'épée ou encore le sacrifice du taureau noir, devient la condition même de sa réalisation. Elle investit le prêtre du pouvoir de communiquer avec les guerriers défunts, à l'instar du sang versé sur leurs tombes. 
On le voit, l'helléniste peine à démêler les préoccupations qui sous-tendent l'élaboration des normes rituelles et à cerner le rôle assigné aux couleurs et aux codes vestimentaires. Nous aurions pu prendre une autre série d'exemples qui a retenu l'attention de nombreux chercheurs : celle des inscriptions réglementant la parure des fidèles souhaitant pénétrer dans le sanctuaire ou participer aux cérémonies (Mills, 1984). Mais dans ce type de textes, adressés prioritairement aux femmes, c'est souvent moins une couleur précise (éventuellement la pourpre ou le vêtement noir) que le fait d'avoir un vêtement teint ou décoré qui est prohibé : les couleurs vives font alors l'objet d'un contrôle, au même titre que les bijoux et les éléments de parure (Grand-Clément, 2011b). Diverses explications ont été avancées : exigences de pureté rituelle se manifestant par une prédilection pour la sobriété vestimentaire (couleurs vs blanc); volonté de limiter l'ostentation des aristocrates (couleurs comme signe de luxe); manifestation du contrôle social exercé par les hommes sur leurs épouses et régulation des émotions dans le cadre des fêtes religieuses (couleurs comme instrument de séduction). On pourrait ajouter surtout qu'il s'agit de créer une communauté de fidèles : la norme vise à renforcer la solidarité du groupe en créant les conditions requises pour faire du rituel une expérience collective et partagée (Culham, 1986). Cela implique notamment une harmonisation chromatique : chacun, le temps du rituel, renonce éventuellement aux couleurs qu'il arbore au quotidien, pour mieux se fondre dans la masse.

Au terme de ce voyage en pays grec, on ne rappellera jamais assez qu'une couleur n'acquiert une signification, une efficacité propre, que lors du déroulement d'un rite donné : les configurations varient et il n'existe pas de code symbolique fixe et général qui régirait l'ensemble des normes cultuelles grecques. Le mode d'action des couleurs doit être appréhendé en termes de relation, de manière dynamique et fluide : elles interagissent les unes par rapport aux autres. Et comme le souligne M. Taussig, ce sont d'abord des matières : eau, laine, huile, pelage des victimes sacrificielles, tissus, qui servent à établir et à entretenir la communication avec les dieux. On touche alors du doigt l'une des limites de l'étude que nous avons esquissée ici. En ne relevant que les références explicites aux couleurs dans les textes, nous avons laissé de côté l'emploi de nombreux éléments et substances comme le lait, le vin, la farine, le miel, les fleurs, et dont la couleur, associée à d'autres propriétés sensorielles, jouait un rôle dans le rituel. C'est surtout le cas du sang, le plus puissant des colorants, qui se caractérise dans l'imaginaire grec par une aptitude à la métamorphose chromatique, indissociable de son pouvoir coagulant. Dans la tradition littéraire, il est tour à tour qualifié de rouge, d'écarlate, de pourpre, de sombre ou de noir. Or, c'est précisément cette aptitude à la métamorphose chromatique qui lui confère son agency (Grand-Clément, 2016). 
Cette dernière remarque offre l'occasion de revenir sur la question posée en introduction. La couleur possède effectivement aux yeux des Grecs une efficacité, mobilisée pour la réussite de certains rituels. Le vocabulaire en témoigne : les Grecs disposent de plusieurs mots pour dire " couleur ", mais lorsqu'ils veulent parler de la couleur dans sa matérialité, pour évoquer pigments et colorants, ils emploient le terme pharmakon. Celui-ci désigne tout type de substance active, qui, manipulée par des mains expertes et suivant un protocole précis, peut agir de manière bénéfique ou nocive - poison ou remède. Entre les mains de magiciennes telles Médée ou Circé, elles deviennent de redoutables moyens d'action : des «substances magiques polymorphes ", à n'en pas douter.

\author{
Adeline GRAND-CLÉMENT \\ Équipe PLH-ERASME \\ Université Toulouse 2 Jean Jaurès \\ adelinegc@yahoo.fr
}

\title{
Bibliographie
}

Brulé Pierre, Mehl Véronique (dirs.), 2008, Le sacrifice antique. Vestiges, procédures et stratégies, Rennes, Presses universitaires de Rennes.

Brulé Pierre, Touzé Rachel, 2008, "Le hiereion, phusis et psuchè d'un medium ", in Brulé P., Mehl V. (dirs.), Le sacrifice antique. Vestiges, procédures et stratégies, Rennes, Presses universitaires de Rennes, p. 111-138.

BRULÉ Pierre, 2009, La norme en matière religieuse en Grèce ancienne, Liège, Centre international d'étude de la religion grecque antique.

Carbon Jan-Mathieu, Pirenne-Delforge Vinciane, 2012, "Beyond Greek "Sacred Laws” ", Kernos, 25, p. 163-182.

CARrin-Bouez Marine, 1978, "La couleur prescrite. Discrimination et accumulation dans le sacrifice santal (Inde) ", in Tornay S. (éd.), Voir et nommer les couleurs, Nanterre, Université de Paris X, p. 581-607.

Chaniotis Angelos, 2012, "Greek Ritual Purity: From Automatisms to Moral Distinctions ", in Rösch P., Simon U. (eds.), How Purity is Made, Wiesbaden, Harrassowitz Verlag, p. 123-139.

CHANKowski Véronique, 2014, "Réparer les idoles, entretenir les lieux de culte ", Tekhnè, 40, p. 42-45.

Chantraine Pierre, 1999, Dictionnaire étymologique de la langue grecque, Paris, Klincksieck.

Culham Patrice, 1986, "Again, What Meaning Lies in Colour! ", Zeitschrift für Papyrologie und Epigraphik, 64, p. 235-245.

Detienne Marcel, Vernant Jean-Pierre, La cuisine du sacrifice en pays grec, Paris, Gallimard, 1979.

Dimakopoulou Adrienne, 2010, Chlôrêis aêdôn, pale rossignol. Une étude sémantique, Paris, Apolis.

Escobar Ticio, 2007, The Curse of Nemur: In Search of Art, Myth, and Ritual of the Ishir, Pittsburgh, University of Pittsburgh Press. 
FEYel Christophe, 2006, "La dokimasia des animaux sacrifiés », Revue de philologie, de littérature et d'histoire anciennes, LXXX, p. 33-55.

GAUTHIER Philippe, 1984, "La dokimasia des victimes. Note sur une inscription d'Entella ", Annali della Scuola Superiore di Pisa, ser. 3, 14.3, p. 845-848.

Georgoudi Stella, 2007, "Quelles victimes pour les dieux ? À propos des animaux "sacrifiables" dans le monde grec ", in Cam M.-C. (dir.), La médecine vétérinaire antique, Rennes, Presses universitaires de Rennes, p. 35-44.

-, 2010, "Comment régler les theia pragmata. Pour une étude de ce qu'on appelle "Lois sacrées” ", Mètis, NS 8, p. 39-54.

GERNET Louis, 1957, "Dénomination et perception des couleurs chez les Grecs ", in Meyerson I. (éd.), Problèmes de la couleur, Paris, SEVPEN, p. 313-326.

GersChel Lucien, 1966, "Couleurs et teinture chez divers peuples indo-européens ", Annales ESC, XXI, p. 608-631.

Grand-ClÉment Adeline, 2011a, La fabrique des couleurs. Histoire du paysage sensible des Grecs anciens (VIII ${ }^{e}$-début du ves. av. n. è.), Paris, De Boccard.

-, 2011b, «Du bon usage du vêtement bariolé ", in Bodiou L., Gherchanoc F., Huet V. et Mehl V. (éds.), Parures et artifices : le corps exposé dans l'Antiquité, Paris, L'Harmattan, p. 255-273.

-, 2016, « Noir, pourpre et écarlate : les propriétés chromatiques du sang dans l'imaginaire grec ", in Bodiou L., Mehl V. (éds.), L’Antiquité écarlate, Rennes, Presses universitaires de Rennes.

Homolle Théophile, 1890, "Comptes et inventaires des temples déliens en l'année 279 », Bulletin de Correspondance Hellénique, p. 389-511.

IRWIN Eleanor, 1974, Colour Terms in Greek Poetry, Toronto, Hakkert.

Jacobson-Widding Anita, 1979, Red-White-Black as a Mode of Thought. A Study of Triadic Classification by Colours in the Ritual Symbolism and Cognitive Thought of the Peoples of the Lower Congo, Uppsala, Almqvist ans Wiksell.

Jones Christopher, 1999, "Processional Colors ", Studies in the History of Art, 56, p. 246-257.

Le Guen-Pollet Brigitte, 1991, La vie religieuse dans le monde grec du ve siècle au ${ }_{I I I}{ }^{e}$ siècle avant notre ère, Toulouse, Presses universitaires du Midi.

Lupu Eran, 2009, Greek Sacred Law: A Collection of New Documents, Leiden, Boston, Brill, 2009.

Mahmood Saba, 2009, Politique de la piété. Le féminisme à l'épreuve du renouvean islamique, [trad. fr. de Politics of Piety, 2005], Paris, La Découverte.

Mills Harriane, 1984, "Greek Clothing Regulation: Sacred and Profane ", Zeitschrift für Papyrologie und Epigraphik, 55, p. 255-265.

Naiden F. S., 2006, Ancient Supplication, Oxford, Oxford University Press.

PARKer Robert, 1983, Miasma: Pollution and Purification in Early Greek Religion, Oxford, Clarendon Press.

-, 2004, "What Are Sacred Laws? ", in Harris E. M., Rubinstein L. (eds.), The Law and the Courts in Ancient Greece, Londres, Duckworth, p. 57-70.

Pastoureau Michel, 1997, Jésus chez le teinturier. Couleurs et teintures dans l'Occident médiéval, Paris, Le Léopard d'or.

Pirenne-Delforge Vinciane, 2008, Retour à la source. Pausanias et la religion grecque, Liège, Centre international d'étude de la religion grecque antique. 
Simon Udo, 2012, "Why Purity? An Introduction », in Rösch P., Simon U. (eds.), How

Purity is Made, Wiesbaden, Harrassowitz, p. 1-37.

SOKOLOWSKI Franciszek, 1955, Lois sacrées de l'Asie Mineure, Paris, De Boccard.

-, 1962, Lois sacrées des cités grecques, Supplément, Paris, De Boccard.

-, 1969, Lois sacrées des cités grecques, Paris, De Boccard.

Stratiki Kerasia, 2004, "Melas in Greek Cultural Practice: The Case of Heroic Sacrifices in the Periegesis of Pausanias ", in Cleland L., Stears K. (eds.), Colour in the Ancient Mediterranean World, Oxford, British Archaeological Reports, p. 106-109.

Taussig Michael, 2009, What Color is the sacred?, Chicago, The Chicago University Press.

VAN STRATEN Folkert T., 1995, Hiera kala: Images of animal sacrifice in archaic and classical Greece, Leiden, Brill.

TORNAy Serge, 2014, Itinéraire nilotique : Afrique orientale, Paris, Karthala.

TuRnER Victor, 1966, "Color classification in Ndembu ritual: A problem in primitive classification ", in Bantono M. (ed.), Anthropological Approaches to the Study of Religion, London, Tavistock, p. 47-84.

VolokHINE Youri, 2010, "Des Séthiens aux Impurs. Un parcours dans l'idéologie égyptienne de l'exclusion ", in Borgeaud Ph., Römer Th., Volokhine Y. (éds.), Interprétations de Moïse. Égypte, Judée, Grèce et Rome, Brill, Leiden-Boston, p. 199-243.

ZaHAN Dominique, 1990, "L'homme et la couleur ", in Poirier J. (dir.), Histoire des moeurs. Tome I : les coordonnées de l'homme et la culture matérielle, Paris, Gallimard, p. $115-180$.

Zografou Athanassia, 2010, "Magic Lamps, Luminous Dreams: Lamps in PGM Recipes ", in Christopoulos M., Karakantza E. D., Levaniouk O. (eds.), Light and Darkness in Ancient Greek Myth and Religion, Plymouth, Lexington Books, p. 276-294.

Zoumbaкi Sophia, 2014, "At the Mercy of Waves and Storms": Roman and Italiote Traders and Settlers in the Cyclades ", in Bonin G., Le Quéré E. (éds.), Pouvoirs, îles et mer. Formes et modalités de l'hégémonie dans les Cyclades antiques, Bordeaux, Ausonius, p. 317-330.

\section{Couleurs, rituels et normes religieuses en Grèce ancienne}

Dans le système de représentations des Grecs anciens, la poikilia ("bigarrure ") était mise en relation avec l'harmonie et la disposition harmonique (disposición armó) $d u$ monde. Mais quel rôle jouaient exactement les couleurs dans les dispositifs rituels, destinés précisément à entretenir de bons rapports avec les puissances divines et maintenir cet ordre cosmique? Les Grecs leur attribuaient-ils une valeur intrinsèque, un mode d'action propre, une forme d'agency? L'étude des inscriptions réglementant les actes du culte et les comportements dans les sanctuaires et lors des rituels révèle que les couleurs peuvent faire l'objet d'une attention particulière dans trois types de cas. D'abord, la blancheur de certaines substances ou matières peut servir à effacer les traces de souillure et à garantir la pureté rituelle au sein de l'espace sacré, pour assurer une communication harmonieuse avec les dieux. Ensuite, la couleur peut être l'une des caractéristiques exigées des animaux sacrifiés aux dieux. Il s'agit souvent de se procurer des bêtes "sans tache ", au pelage uniforme, tantôt blanc, tantôt noir, plus rarement roux. Enfin, la couleur et le décor des vêtements du prêtre et des 
fidèles font l'objet d'une réglementation parfois stricte. Il s'agit alors de distinguer, de hiérarchiser les différents acteurs en fonction de leur statut social et de leur degré de participation aux rituels, mais aussi de garantir l'efficacité des rites.

Mots-clés : couleurs, religion grecque, réglementation, rituels, blancheur.

\section{Colors, rituals and religious norms in ancient Greece}

In Ancient Greece, the notion of poikilia, "colorfulness, variegation", was equated with harmony, and a pleasing arrangement of the world. But what kind of role did colors play in the ritual devices whose function was precisely to maintain good relationships with the divine powers as well as with the cosmic order? Did Greek people see in them an intrinsic value, a specific mode of action, a form of agency? A study of the inscriptions regulating cultic actions and behaviors in the sanctuaries and during rituals reveals that colors can be given a particular attention in three type of cases: first, the whiteness of some substances or materials can serve to erase traces of stains and to guarantee ritual purity within a sacred space, in order to insure a harmonious communication with the gods. Second, color can be one of the characteristics required from the animals sacrificed to the gods. It is often about getting animals "without blemish", with a uniform fur or skin, sometimes white, sometimes black, rarely red. Lastly, the color and the decoration of the clothing of the priest and the worshippers are the object of a regulation that is sometimes strict. The purpose is therefore to distinguish and hierarchize the various actors, depending on their social status and their degree of participation to rituals, but also their ability to guarantee the efficiency of rituals.

Key words: colors, Greek religion, regulation, rituals, whiteness.

\section{Colores, rituales y normas religiosas en la Grecia antigua}

En el sistema de representaciones de la Grecia antigua, la poikilia ("mescolanza de colores y formas") estaba relacionada con la armonía y la disposición armónica del mundo. ¿Pero qué rol jugaban exactamente los colores en el orden cósmico? ¿Le atribuían los Griegos un valor intrínseco, un modo de acción propio, una forma de agency? El estudio de las inscripciones que reglamentan los actos del culto y el comportamiento en los santuarios durante los rituales revela que los colores podían ser objeto de una atención particular en tres tipos de casos. En principio, la blancura de ciertas sustancias o materias puede servir para borrar las huellas de suciedad y para certificar la pureza del ritual en el espacio sagrado, para garantizar una comunicación armoniosa con los dioses. Luego, el color puede ser una de las características exigidas a los animales sacrificados a los dioses. Se trata a menudo de buscar animales "sin mancha”, de pelaje uniforme, ya sean blancos, negros o más raramente rojizos. Finalmente, el color y la decoración de las vestimentas del sacerdote y de los fieles son objeto de una reglamentación a menudo estricta. Se trata entonces de distinguir, de jerarquizar los distintos actores en función de su estatuto social y de su grado de participación en los rituales. Pero también de garantizar la eficacia de los ritos.

Palabras clave: colores, religión griega, reglamentación, rituales, blancura. 
